\title{
The Policy-Driven Enhancement to Reduce Adult Inpatients' Pain Intensity of "No More Than Mild Pain" During the COVID-19 Pandemic
}

\author{
Litaba Efraim Kolobe ${ }^{1, *}$, Nabeeha Tashkandi ${ }^{2}$, Angela Tracy Caswell ${ }^{3}$, Sheikha Al Anizi ${ }^{4}$, \\ Aisha Al Khanbaie ${ }^{1}$, Waad Maringah ${ }^{1}$, Kawther Ajarmeh ${ }^{1}$, Eman Al Ruwaili ${ }^{4}$ \\ ${ }^{1}$ Department of Nursing Pain Management, King Abdulaziz Medical City Hospital, Riyadh, Saudi Arabia \\ ${ }^{2}$ Department of Nursing Administration, King Abdulaziz Medical City Hospital, Riyadh, Saudi Arabia \\ ${ }^{3}$ Department of Nursing Administration, King Abdullah Specialised Children's Hospital, Riyadh, Saudi Arabia \\ ${ }^{4}$ Department of Nursing Pain Management, King Abdullah Specialised Children's Hospital, Riyadh, Saudi Arabia
}

Email address:

kolobe66@yahoo.com (L. E. Kolobe)

*Corresponding author

\section{To cite this article:}

Litaba Efraim Kolobe, Nabeeha Tashkandi, Angela Tracy Caswell, Sheikha Al Anizi, Aisha Al Khanbaie, Waad Maringah, Kawther Ajarmeh, Eman Al Ruwaili. The Policy-Driven Enhancement to Reduce Adult Inpatients' Pain Intensity of "No More Than Mild Pain" During the COVID-19 Pandemic. American Journal of Nursing Science. Vol. 10, No. 5, 2021, pp. 237-243. doi: 10.11648/j.ajns.20211005.14

Received: September 19, 2021; Accepted: October 5, 2021; Published: October 12, 2021

\begin{abstract}
Context: Moderate to severe pain has a profound negative impact on functioning and quality of life, hence nursing guidelines were needed as the driving force to optimize pain nursing interventions. Aim: To enhance the reduction of adult inpatients' pain intensity of not more than mild pain during the COVID-19 pandemic nursing care settings. Methods: For this hospital-wide pain management quality improvement initiative, quarterly cross-sectional pain prevalence surveys were conducted between February 2020 and February 2021. For patients with pain scores less than 4/10 only section one of the survey questionnaires was used, while those with pain scores more than mild pain chart review was performed utilizing section two of the questionnaire. The $80 / 20$ principle was adopted to identify the area that needs attention for specific improvement actions of each unit that achieved less than $80 \%$ of patients with pain intensity less than $4 / 10$. Results: University Hospital A achieved an average of $92 \%$ throughout the four quarters, and $87.4 \%$ was for University Hospital B. Less than $20 \%$ of patients during the four quarters of the surveys had moderate to severe pain in both hospitals. Conclusions: Both two hospitals achieved the target goal of more than $80 \%$ pain prevalence scores of not more than mild pain during the four quarters of the studies. It is therefore strongly recommended that healthcare organizations put in place policies and procedures that assign the nurses the target goal of effective pain management.
\end{abstract}

Keywords: Policies and Procedures, Pain Intensity, No Pain, Mild Pain

\section{Introduction}

Universally pain is a subjective experience that predominantly making people seek help from health care institutions. According to the revised definition of pain by the International Association for the Study of Pain (IASP) after four decades from 1979 to January 2020, states that pain "is an unpleasant sensory and emotional experience associated with or resembling that associated with actual tissue damage" [1-3]. This new definition coincides with the pattern of knowing of the emancipation for reconceptualization to pain knowledge that shifted from perceptions that pain was mainly associated with tissue damage without considering the type of pain. This transformation of knowledge is also parallel with the understanding that pain is multidimensional that encompasses sensory, emotional, cognitive, and social components. The plethora of pain studies indicated that pain is a personal experience considered as a biopsychosocial phenomenon that occurs in the absence of tissue damage or any likely pathophysiological cause $[4,5]$. In this essence, studies have demonstrated that pain is always a personal experience, is 
influenced to varying degrees by biological, psychological, and social factors; pain and nociception are different phenomena; cannot be inferred solely from activity in sensory neurons; and that it is through their life experiences for individuals learn the concept of pain [3]. It is further indicated that pain perception, tolerance, and threshold vary widely from one individual to another, therefore it is difficult to standardize the level of pain for all together [6].

The study took place in two university hospitals in Riyadh, Kingdom of Saudi Arabia, University Hospital A (UHA) and University Hospital B (UHB) accredited by Joint Commission International (JCI) and the Saudi Central Board for Accreditation of Healthcare Institutions (CBAHI). The surveys formed part of the pain management nursing quality improvement key performance indicators aimed to optimize pain management by enhancing all adult inpatients' pain rating score to be less than $4 / 10$ despite their types of pain. It was part of the pain nursing interventions policy and procedure to reduce all inpatients' pain to "patient-reported minimal acceptable" threshold or tolerance outcome of pain intensity of not more than mild pain. In their study finding Carragee and Cheng 2010 indicated that a large majority of patients with spondylolisthesis and degenerative disc disease indicated that the minimum acceptable outcomes included at least a decrease in pain intensity to $3 / 10$ or less and satisfaction for achieving improvements [7]. Pain inappropriately managed results in a negative impact on patient outcomes such as prolonged hospitalization, delayed discharge time, increased hospital costs, health-related issues, poor quality of life therefore it is essential to assess and manage pain to acceptable levels of the patients to enhance patient satisfaction and patient outcome [8]. To achieve optimal pain management and improve patient outcomes, the nursing pain interventions guidelines assigned the fundamental practice of all nurses to perform adequate pain assessment with the intended goal of maintaining pain severity less than 4/10 [9].

As part of a paradigm shift, the profession of nursing has been on the front line of pain assessment and management of different types of pain [10]. This also includes nurses' legal and ethical responsibility to have knowledge, skills, and attitude of understanding that patient pain experience is individualized and must be respected as their rights to ensure comfort and always caring while under care by providing adequate pain management. For ensuring patient safety, the two hospitals' nursing pain interventions were driven by pain nursing policy and procedures that authorized nurses to safeguard that patients' pain intensity was reduced to mild (1$3 / 10)$ or no pain $(0 / 10)$. These nursing guidelines also included: empowering the patient and family members about options available for pain management; use of comprehensive assessment guide tools such as WILDA (words, intensity, location, duration, aggravating and alleviating factors) and pain behavioral indicators; two-hourly as minimal assessment of pain; indicating the type of pain; ensuring use of multimodal pain interventions that include nonpharmacological and pharmacological interventions that quantify levels of pain, and reassessment of pain for effectiveness and adverse effects for pain nursing interventions provided.
Measuring pain with a unidimensional scale such as the Numeric Rating Scale or multidimensional such as FLACC (Face, legs, arms, crying, and consolability) may be used [11]. To evaluate pain intensity acceptable if there was a reduction in a pain score of itself only is considered a gap that may not equate to an improvement in the patient's experience unless incorporating minimal clinical importance difference (MCID) and patient acceptable symptom state (PASS), [12]. The pain experts have suggested that the use of unidimensional pain rating scales only has limitations to provide a comprehensive pain assessment to patients' acceptable symptom states as pain is multidimensional [13].

Pain management nursing intervention guidelines were put into place as resources to guide nurses on the assessments of pain, nursing interventions, pain reassessments after interventions, and standardized pain levels of less than $4 / 10$ as a target goal of maintaining and managing pain. The observation in these two hospitals was that majority of the adult inpatients experience uncontrolled pain of moderate to severe levels regardless of either acute or chronic pain. Even though is well known that pain management should be guided by some measures of patient-reported severity of their longterm condition, the aim was more to optimize pain management parallel to acute pain management by establishing a level of "no more than mild pain" as the target goal for pain nursing interventions [14]. The pillar of achieving this goal was based on education provided to nurses during pain management workshops and unit in-service training with the content that includes: that no matter the patients experienced the pain of not more than mild pain they must continue with pain assessments as per guidelines, to continue with nonpharmacological interventions, regular and breakthrough analgesia as part of pharmacological interventions. In addition, they were also educated to consider the multidimensional nature of pain that could lead to human suffering and disability, to respect the subjectivity of pain intensity, to reassess the effect of pain treatment, to manage patients as individuals by considering their pain threshold, pain tolerance, and dependence to pain medications (Higgins et al., 2019) $[14,15]$. The outcome of pain management applied to all adult inpatients was monitored in these two hospitals by approving the hospital-wide key performance indicator of conducting quarterly pain prevalence. The aim was to determine if patients were experiencing uncontrolled pain while receiving care [16]. This was also to ensure that pain management was optimized for all hospitalized patients.

To assess pain intensity in patients who can self-report their pain, either the Wong-Baker FACES pain scale (WBF), or the Numeric Rating Scale (NRS) was used. In addition, the FLACC pain scale was used to assess those patients who could not verbalize their pain or were cognitively impaired. Despite that FLACC was originally developed as a pediatric pain scale, it has been shown in several studies to have acceptable validity and reliability for use in adults who could not self-report their pain [11]. This behavioral pain scale was used by observing the patient in 5 minutes for pain behavioral indicators such as facial expressions, 
vocalizations, and body movements, rating the five categories on the score of 0 to 2 scale, adding the scores together for a total possible score of 0 to 10 [17]. The three used pain scales' cutoff pain rating was 0 to $10(0=$ no pain, 1 $3=$ mild pain, $4-6=$ moderate pain, and $7-10=$ severe pain $)$.

Pain assessment in these two hospitals was considered as the first step in the pain management process that relies on a patient's self-report well known as the golden standard of pain assessment. This is the most accurate and reliable evidence of the existence of pain and its severity, for patients of all ages, regardless of communication or cognitive impairments $[10,18]$. Conventionally to standardize the pain experience of patients through pain score, need to define the pain intensity categories or description of the type of pain used to define and quantify the score as $0=$ no pain, $1-3=$ mild, $4-6=$ moderate, and $7-10=$ severe $[18,19]$. It is recommended that pain management should be guided by some measures of patient-reported severity and the type of pain such as chronic pain management or for example in acute pain management, a level of "no more than mild pain" can be established as a treatment goal [14]. Hence the two University hospitals' pain nursing interventions were guided by patient-reported pain severity ratings aimed to achieve a measurable pain intervention goal of less than $4 / 10$ for all adult inpatients despite their type of pain that may include either nociceptive, neuropathic, or nociplastic pain [20].

There are many experiences to tell from patient perspectives related to exacerbation of pain intensity during the unprecedented crisis of coronavirus disease 2019 (COVID-19) since 2020, either as COVID-19 related disease processes or other diseases related to pain symptoms that have impacted their lives and health of persons worldwide hence the recommendation is to consider many factors contributed to a potential increase in chronic pain after COVID 19 pandemic [20]. It was therefore the motivation of the two university hospitals to downstream the negative impact of uncontrolled pain and COVID-19 related consequences that may aggravate the prevalence of chronic pain [20]. In this context, this motivational factor influenced the two hospitals to initiate hospital-wide standardization of patients' pain severity to be considered acceptable if not more than mild pain despite individualization of pain experiences and type of pain.

Moderate (4-6/10) to severe (7-10/) pain have a profound negative impact on patients functioning and quality of life, hence the pain nursing guidelines stipulated nursing interventions that recommended the category of pain intensity of not more than mild from pain scales a clear cutoff with pain ratings [21]. The cutoff of pain ratings of pain scales used to measure pain levels in these two hospitals was 0 to 10 . Hence pain intensity of 4-10/10 suggested a significant problem that needs attention. [21]. To our knowledge, there is no existing quality monitoring initiative study with the core objective of conducting quarterly pain prevalence to determine whether adult inpatients were experiencing uncontrolled pain while receiving care, and that aimed to enhance reduction of their pain severity to no more than mild pain experience. In this article, the purpose is to describe the outcomes of prevalence surveys during the COVID 19 pandemic in the context of the University Hospital A and B, Riyadh, Kingdom of Saudi Arabia intended to enhance the reduction of adult inpatients' pain severity of not more than mild pain, between February 2020 and February 2021.

\section{Methods}

These pain prevalence studies were approved by nursing leadership as part of hospital-wide key performance indicators for pain management quality improvement at two Saudi Arabian university hospitals in Riyadh City between February 2020 and February 2021. Cross-sectional pain prevalence surveys were conducted quarterly. The goal was to survey all adult inpatients on a scheduled day per calendar planned by nursing standards of care to determine if all patients while receiving care their pain was reduced to mild or no pain. The purpose was to measure the number of patients that experience uncontrolled pain while receiving care targeting to optimize the pain management of all hospitalized patients.

Quarterly, a day was chosen to provide a snapshot of pain experience by screening pain, starting the study from 08:00 to 12:00 to all units of adult patients included and excluded adult patients in operating rooms, labor wards, adult patients in post-anesthetic care units, ambulatory care, hemodialysis patients, adult patients for procedures, emergency care patients still with emergency status. To prepare for data collection, mandatory training was provided by Pain Team Nurses (PTNs) a day before the prevalence day to the nurse managers and clinical resource nurses about the data collection methods, use of pain assessment tools (WILDA, FLACC, Wong-Baker FACES Scale and Numeric rating scale), and how to to use the survey tool.

For the population size, the two hospitals have approximately 1800 bed capacity. Patients and family members were also informed about the prevalence study a day before the study day. All units with patients classified as adults were listed on the inpatient census at 08:00 hours to participate in the surveys.

During the day of the pain prevalence survey, the surveyors were randomly allocated by the pain team to assess the patients to the different units. All the rights of the patients were ethically considered for inclusion and exclusion criteria mentioned.

Data was collected using a structured survey questionnaire developed by the pain team and pretested for validation and reliability. The survey tool contained 17 items of closedended questions and was divided into two sections.

In section one (1) of the questionnaire: was for interviewing the patient pain experience (self-report of pain) or observing the patient behavioral pain indicators (proxy-reported pain), to obtain the information on the patient's demographics, pain scale used, and responses of verbal patients to indicate if the nurses were showing them pain scales during pain assessment. Pain rating scales such, as the FLACC pain scale, Wong-Baker FACES pain rating scale, or Numeric Rating Scale was utilized to measure the pain intensity. Each adult patient unit was 
mandated to not have more than $20 \%$ of the total number of patients assessed experiencing pain more than 3/10 if this occurs an action plan was to be implemented by such unit to improve the pain management as part of optimizing pain management of patients receiving care. The target goal was to achieve $80 \%$ of patients for each unit participating in the survey to have patients' pain reduced to mild or no pain. A pain score above $4 / 10$ for each unit was only acceptable if it was less than $20 \%$ of the total number of patients in that unit.

In section two (2) of the questionnaire was a chart review of only those patients who experienced a pain score of more than $3 / 10$. The electronic medical records were audited to rule out the contributing factors. The data collectors audited if there was documentation of pain intensity for the last two cycles of pain experienced, pain assessment guide tool (WILDA) used, the frequency of pain assessment for the last 24 hours, type of pain, use of multimodal analgesia, use of non-pharmacological interventions, if the analgesia were prescribed according to the level of pain, was pain reassessed as per pain nursing guidelines, and whether was pain education offered to the patient.

For data entry and analysis, Microsoft 365 Excel worksheet was used. For prioritization of the outcome of the surveys, the $80 / 20$ principle was adopted that any unit that achieved less than $80 \%$ of patients with pain prevalence scores of more than mild pain, warranted the PTNs attention to focus on attributable problems [22] by collaborating with such units for improvement initiatives. The target goal was to achieve $80 \%$ as cut offline for each unit participating in the survey to have patients' pain reduced to mild or no pain. A pain score above $4 / 10$ for each unit was only acceptable if it was less than $20 \%$ of the total number of patients in that unit.

Only the findings of section one of the prevalence surveys between February 2020 and February 2021 are presented and discussed below.

\section{Results}

The quarterly completed surveys were returned by the surveyors for analysis and the results presented in this manuscript are related to the outcomes of phase one of the pain prevalence surveys contacted between February 2020 and February 2021

No pain (0/10) and mild pain (1-3/10) prevalence pain scores of patients in UHA are as illustrated in Figure 1, while for patients in UHB are in Figure 3. The uncontrolled pain severity pain scores considered as moderate (4-6/10) to severe (7-10/10) are portrayed in Figure 2 for UHA and Figure 4 for UHB.

The UHA adult inpatients' pain severity as portrayed in Figure 1 indicates that in February 2020 the average pain prevalence scores were $93.3 \%(\mathrm{n}=588 ; \mathrm{N}=630)$ for no pain $(\mathrm{f}=77,9 \% ; \mathrm{F}=491)$ and mild pain $(\mathrm{f}=15,4 \% ; \mathrm{F}=97)$. This finding is for 41 out of 46 units that achieved more than $80 \%$ of patients with pain not more than mild pain. The May prevalence was omitted due to surged number of patients COVID-19 pandemic. Figure 1 illustrates the pain severity scores for prevalence in September 2020 was $91,3 \% \quad(n=548 ; N=600)$ for no pain $(\mathrm{f}=77,3 \% ; \mathrm{F}=464)$ and mild pain $(\mathrm{f}=14.0 \% ; \mathrm{F}=84)$. This was from 44 out of 46 units that achieved more than $80 \%$ of patients with no more than mild pain. In November 2020 out of 46 units, only 41 achieved more than $80 \%$ of patients with pain scores of less than $4 / 10$. The average for pain not more than mild was $92,0 \%(\mathrm{n}=581 ; \mathrm{N}=631)$, for no pain $(\mathrm{f}=79,0 \% ; \mathrm{F}=499)$ and mild pain $(\mathrm{f}=13,0 \% ; \mathrm{F}=82)$. During the first quarter in February 2021, only five out of 46 units achieved less than $80 \%$ of patients with pain of not more than $4 / 10$. This means for controlled pain, the average was $92,0 \%(\mathrm{n}=566 ; \mathrm{N}=615)$ for no pain $(\mathrm{f}=81,0 \%$; $\mathrm{F}=498)$ and mild pain $(\mathrm{f}=11,0 \% ; \mathrm{F}=68)$. The total average prevalence scores of the four studies were $92 \%$ of pain scores not more than mild pain.

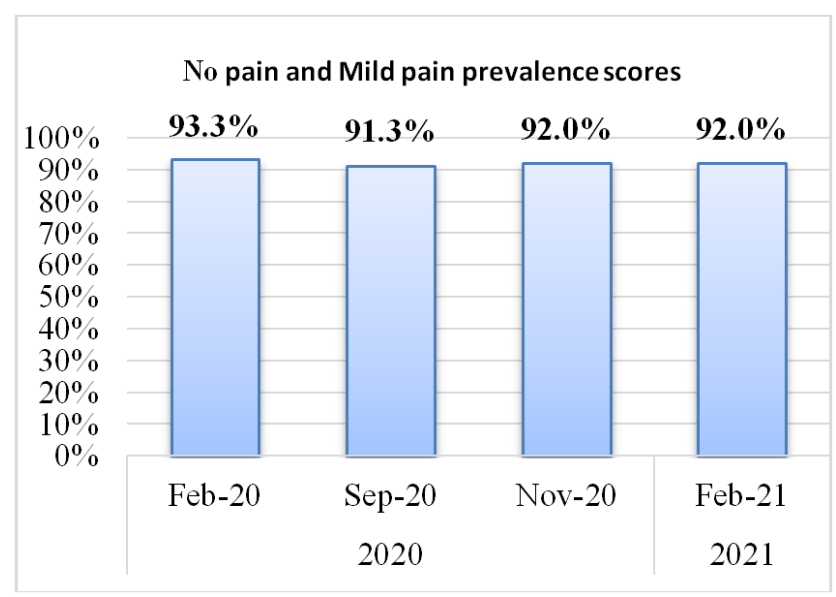

Figure 1. UHA no pain and mild pain scores.

In essence, throughout any of the four prevalence studies, not more than 5 units achieved less than $80 \%$ of patients with more than mild pain. Figure 2 portrays the uncontrolled pain of the lowest number of patients with a pain score of more than $3 / 10$ that was in February 2020 with 42 patients $(\mathrm{f}=6.7 \% ; \mathrm{n}=42 ; \mathrm{N}=630$ ), while the highest number of patients with a pain score of more than $3 / 10$ was in February 2021 with 51 patients $(\mathrm{f}=8.0 \% ; \mathrm{n}=51 ; \mathrm{N}=615)$.

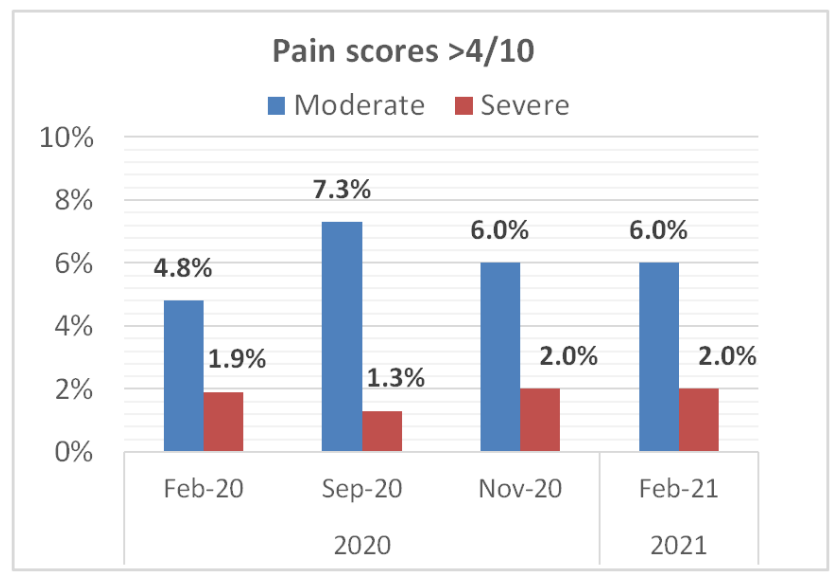

Figure 2. UHA moderate pain and severe pain scores.

This implies that despite the COVID-19 pandemic nursing care situations, the pain of adult inpatients was highly 
controlled during the four quarters. The findings demonstrate that even despite the highest number of patients in February 2021 , the units sustained an average of $92 \%$ of patients with pain scores of no more than mild pain previously achieved in November 2020. Significantly the findings illustrate UHA achieved the target goal of $80 \%$ as a minimally acceptable average pain prevalence score of no more than mild pain throughout the four surveys regardless of the COVID-19 pandemic nursing care situations.

In UHB, the adult inpatients' pain prevalence average scores of less than 4/10 conducted in February 2020 was $86,1 \%(\mathrm{n}=93 ; \mathrm{N}=108)$ for no pain $(\mathrm{f}=68,5 \% ; \mathrm{F}=74)$ and mild pain $(\mathrm{f}=17,6 \% ; \mathrm{F}=19)$ (see Figure 3 ).

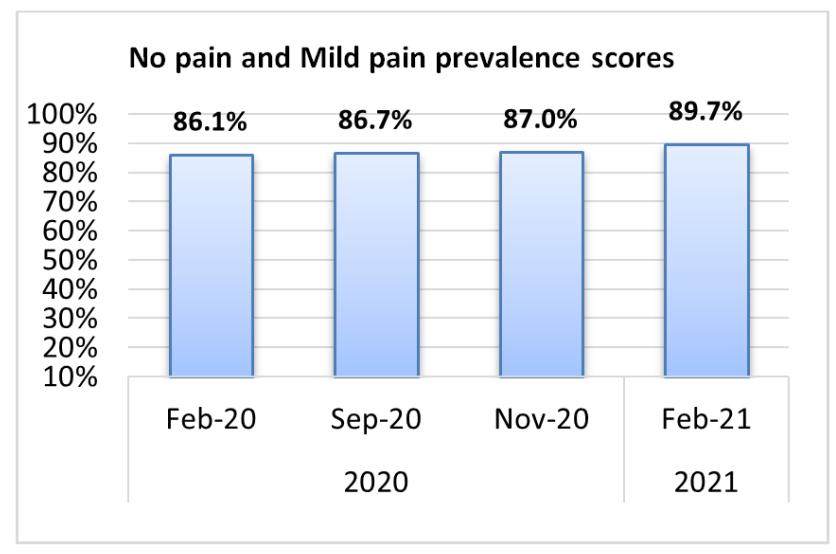

Figure 3. UHB no pain and mild pain scores.

It was seven units out of eight units that achieved more than $80 \%$ of patients with no more than mild pain. The May prevalence was also not carried out in this hospital due to risen number of patients with the COVID-19 pandemic. The average pain severity scores for prevalence in September 2020 was $86,7 \%(\mathrm{n}=98 ; \mathrm{N}=113)$ for no pain $(\mathrm{f}=77,0 \%$; $\mathrm{F}=87$ ) and mild pain ( $\mathrm{f}=9,7 \% ; \mathrm{F}=11)$. Six out of eight units managed to achieve more than $80 \%$ of patients with pain severity not exceeding mild pain $(<4 / 10)$. In November 2020 the average score was $87.0 \%(\mathrm{n}=107 ; \mathrm{N}=123)$ for no pain $(\mathrm{f}=76,0 \% ; \mathrm{F}=93)$ and mild pain $(\mathrm{f}=11,0 \% ; \mathrm{F}=14)$. Only five units out of eight achieved more than $80 \%$ of patients with pain scores of less than 4/10. The first quarter in 2021 February only six units out of eight achieved more than $80 \%$ of pain scores less $4 / 10$ with the average pain prevalence scores of $89,7 \%(\mathrm{n}=96 ; \mathrm{N}=107)$ for no pain $(\mathrm{f}=75,7 \% ; \mathrm{F}=81)$ and mild pain $(\mathrm{f}=14,0 \% ; \mathrm{F}=15)$. The total average pain prevalence scores of the four studies were $87.4 \%$ with pain not more than mild pain. In essence, during any of the four prevalence studies, not more than 3 units achieved less than $80 \%$ of patients with more than mild pain. As portrayed in Figure 4, the lowest number of patients with a pain score of more than $3 / 10$ was in February 2021 with 11 patients $(\mathrm{f}=10,3 \% ; \mathrm{n}=11 ; \mathrm{N}=107)$ and the highest number of patients with a pain score of more than $3 / 10$ was in February 2020 with 15 patients $(\mathrm{f}=13,9 \% ; \mathrm{n}=15 ; \mathrm{N}=108)$.

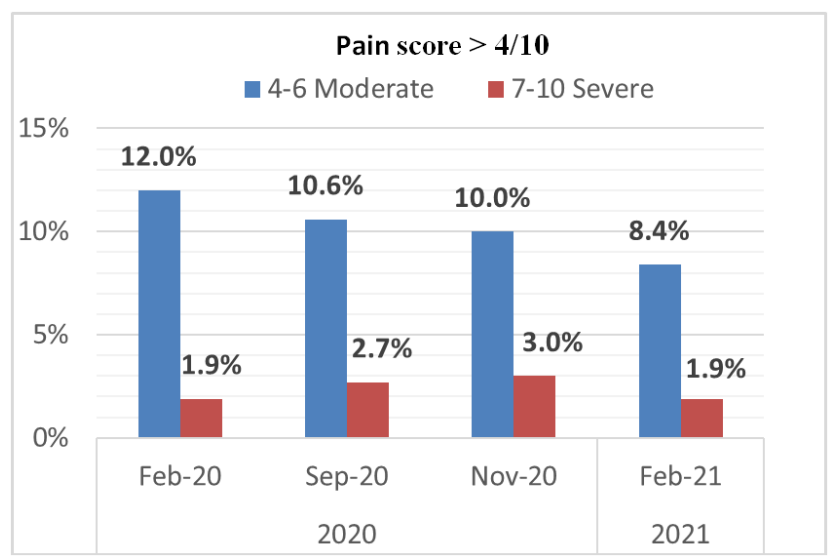

Figure 4. UHB moderate and severe pain scores.

Regardless of the COVID-19 pandemic, the pain of adult inpatients was highly controlled for the duration of the four quarters more especially the last quarter of the pain prevalence surveys. Suggesting that the patients' pain was mostly controlled in February 2021 with an average of 89,7\% of patients with pain scores of not more than mild pain. This demonstrates that UHB also accomplished the target goal of $80 \%$ as a minimally acceptable average pain prevalence score of not more than mild pain through the four surveys despite the COVID-19 pandemic. The nursing policy and procedures for pain interventions were the motivation factor that enhanced patients' pain experiences of not more than mild pain $(0$ to $3 / 10)$.

\section{Discussions}

The present combined pain prevalence surveys are designed to indicate how to reduce adult inpatients' pain intensity of "no more than mild pain" during the COVID-19 pandemic despite their types of pain. The hospital-wide pain prevalence surveys conducted in four quarters formed part of the key performance indicators that played a major role in continuous quality improvement outcomes that enhanced change strategies in pain management and data analysis that supported the implementation of the process of these surveys [23]. The availability of pain nursing guidelines enhanced the assigned nursing practice within these two-hospital systems as a reference that standardized the implementation of this pain management quality improvement initiative. To achieve the target goal of nursing interventions was based on these two hospitals' pain management nursing guidelines that stipulated to all inpatient units to maintain pain of not more than mild pain. The adoption of the $80 / 20$ principle for the outcome of achieving $80 \%$ of patients with pain levels of not more than mild pain was the driving force that synergized the effectiveness of achievement and sustainability of this target goal despite the COVID-19 pandemic.

Studies recommended that health care institutions must use the principles of quality improvement to demonstrate appropriate assessment and effective management of pain, and guidelines as adopted in these two hospitals [24, 25]. Despite other studies that indicated institutions using guidelines only had 
success in improving pain assessment rates, and not sustaining reduction of pain, was not a case in these surveys [24]. The application of the 80:20 principle to these pain prevalence surveys included achieving a goal of maintaining pain less than $4 / 10$ in all inpatient units regardless of the types of pain patients experienced. Hence the expected outcomes of achievements for each unit were not more than $20 \%$ of patients with pain scores of more than mild pain. The chart review of all patients with pain of more than $4 / 10$ was utilized to rule out the contributory factors of uncontrolled pain that enabled the engagement of such units in unit-based quality improvement initiatives focusing on the identified care loopholes.

Reducing adult inpatients' pain intensity of "no more than mild pain" during the COVID-19 pandemic as was with successful outcomes during the four quarters the surveys were conducted even though individual patients experienced different types of pain. In each survey, the average number of patients exceeded $80 \%$ of pain intensity of not more than mild pain that was considered satisfactory to complete effect of pain management. Few units were also identified during this COVID-19 pandemic, and this enabled the PTNs to collaborate with those units to influence pain management optimization from unit-based settings. In this principle, the audited files enabled the units to focus on the area that needed improvements.

The findings of these pain nursing management improvement initiatives demonstrated that UHA attained the total average pain prevalence scores of $92 \%$ for pain severity not more than mild pain throughout the four studies, whereas in UHB the average was $87.4 \%$. Both hospitals maintained controlled pain levels within the acceptable concept of the 80:20 principle of quality improvement initiative.

The shared findings demonstrated a great success of these two university hospitals on how the policy enhanced reductions of adult inpatients' pain intensity of "no more than mild pain" during the COVID-19 pandemic. This effectiveness of inpatients' reduced pain severity outcomes was suggestive of possible improved patient satisfaction and sign of patient acceptable symptom state during hospitalization. These pain initiatives also directly optimized the pain management as nurses were guided by the nursing guidelines' steps to follow for each level of pain that patients experienced for the choice of interventions implemented.

One significant limitation of this pain quality improvement initiative indicated that not only pain severity is the measurement of improving individual patient's pain severity, but to considers pain as a biopsychosocial phenomenon and is impacted by various factors. In addition, types of pain also play a major part in controlling pain effectively. Despite the identified limitations, the availability of resources such as nursing pain intervention guidelines and application through nursing practice demonstrated effectiveness in acute patient settings enhancing the reduction of adult inpatients' pain intensity to an acceptable state of not more mild pain.

\section{Conclusion}

In the present surveys, the use of policies and procedures of pain nursing interventions enhanced the reduction of patients' pain severity not more than mild pain during COVID-19 pandemic care settings. It is significant and strongly recommended in this study context that healthcare organizations put in place policies and procedures controlling the pain nursing interventions that guide the nurses on how to effectively manage all patients with different types of pain using pain tools with cutoffs of pain ratings. It is also important to indicate in the future information sharing the factors that contributed to the pain of more than mild pain identified in section two of chart reviews conducted. In this essence, further monitoring is essential to assess the effectiveness of pain nursing intervention guidelines to individual patient's acceptable symptom states of the pain experience. We hope that this endeavor to reduce inpatients' pain intensity may optimize the pain management in the context of other institutions as proven successful in these two hospital settings.

\section{Conflict of Interest Statement}

The authors declare that they have no competing interest.

\section{Acknowledgements}

We would like to thank our Nursing Administration for their valuable advice and guidance during these prevalence surveys. The authors would like to thank the Nurses of the two hospitals for prioritizing pain management and Surveyors who participated during the prevalence studies. We also thank Felcor May Penurabia for always analyzing the data collected.

\section{References}

[1] McDermid, J, C., Donner, A., Richards, R. S., \& Roth, J. H. (2002). Patient versus injury factors as predictors of pain and disability six months after a distal radius fracture. Journal of Clinical Epidemiology, 55, 849-854.

[2] Raja, S. N., Carr, D. B., Cohen, M., Finnerup, N. B., Flor, H., Gibson, S., Keefe, F. J., Mogil, J. S., Ringkamp, M. \& Sluka, K. A. J. P. (2020). The revised International Association for the Study of Pain definition of pain: concepts, challenges, and compromises. 161, 1976-1982.

[3] Malik, N. A. J. A., Pain \& Care, I. (2020). Revised definition of pain by 'International Association for the Study of Pain': Concepts, challenges and compromises. 24, 481-483.

[4] Cohen, M., Quintner, J., \& Van Rysewyk, S. J. P. R. (2018). Reconsidering the International Association for the Study of Pain definition of pain. 3 .

[5] Tesarz, J., \& Eich, W. J. P. (2017). A conceptual framework for "updating the definition of pain". 158, 1177-1178.

[6] Adem, A. O., Obsa, M. S., Worji, T. A. J. I. J. O. M., \& Sciences, M. (2017). Assessments of health professionals' knowledge towards post-operative pain management in Asella Teaching and Referral Hospital, South East Ethiopia. 9, 137-141. 
[7] Carragee, E. J. \& Cheng, I. J. T. S. J. (2010). Minimum acceptable outcomes after lumbar spinal fusion. 10, 313-320.

[8] Vitullo, M. (2020). The Clinically Aligned Pain Assessment Tool (CAPA) Evidenced-Based Change Project-Benchmark Study.

[9] Kang, Y., \& Demiris, G. J. I. J. O. O. P. N. (2018). Self-report pain assessment tools for cognitively intact older adults: Integrative review. 13, e12170.

[10] Booker, S. Q., Herr, K. A., \& Horgas, A. L. J. P. M. N. (2020). A paradigm shift for movement-based pain assessment in older adults: Practice, policy and regulatory drivers.

[11] Varndell, W., Fry, M., \& Elliot, D. J. J. O. C. N. (2017). A systematic review of observational pain assessment instruments for use with nonverbal intubated critically ill adult patients in the emergency department: an assessment of their suitability and psychometric properties. 26, 7-32.

[12] Myles, P., Myles, D., Galagher, W., Boyd, D., Chew, C., Macdonald, N. \& Dennis, A. J. B. B. J. O. A. 2017. Measuring acute postoperative pain using the visual analog scale: the minimal clinically important difference and patient acceptable symptom state. $118,424-429$.

[13] Jumbo, S. U., Macdermid, J. C., Packham, T. L., Athwal, G. S., Faber, K. J. J. H., \& Outcomes, Q. O. L. (2020). Reproducibility: reliability and agreement parameters of the Revised Short McGill Pain Questionnaire Version-2 for use in patients with musculoskeletal shoulder pain. 18, 1-12.

[14] Treede, R.-D., Rief, W., Barke, A., Aziz, Q., Bennett, M. I., Benoliel, R., Cohen, M., Evers, S., Finnerup, N. B., \& First, M. B. J. P. (2019). Chronic pain as a symptom or a disease: the IASP Classification of Chronic Pain for the International Classification of Diseases (ICD-11). 160, 19-27.

[15] Higgins, C., Smith, B. \& Matthews, K. J. B. J. O. A. (2019). Evidence of opioid-induced hyperalgesia in clinical populations after chronic opioid exposure: a systematic review and meta-analysis. 122, e114-e126.

[16] Taylor, E. M., Boyer, K., Campbell, F. A. J. P. R., \& Management (2008). Pain in hospitalized children: a prospective cross-sectional survey of pain prevalence, intensity, assessment and management in a Canadian pediatric teaching hospital. 13, 25-32.

[17] Crellin, D. J., Harrisson, D., Sanstamaria, N., Huoque, H., \& Babl, F. E. J. T. J. O. P. (2018). The psychometric properties of the FLACC scale used to assess procedural pain. 19, 862-872.

[18] Tsze, D. S., Hirschfeld, G., Dayan, P. S., Bulloch, B., \& Von Baeyer, C. L. J. P. E. C. (2018). Defining no pain, mild, moderate, and severe pain based on the Faces Pain ScaleRevised and Color Analog Scale in children with acute pain. 34, 537.

[19] Karcioglu, O., Topacoglu, H., Dikme, O., \& Dikme, O. J. T. A. J. O. E. M. (2018). A systematic review of the pain scales in adults: which to use? $36,707-714$.

[20] Clauw, D. J., Häuser, W., Cohen, S. P., \& Fitzcharles, M.-A. J. P. (2020). Considering the potential for an increase in chronic pain after the COVID-19 pandemic. 161, 1694.

[21] Miro, J., De La Vega, R., Sole, E., Racine, M., Jensen, M. P., Galan, S., Engel, J. M. J. D. \& Rehabilitation. (2017). Defining mild, moderate, and severe pain in young people with physical disabilities. 39, 1131-1135.

[22] Williams, E. S., Savage, G. T., Patrician, P. A. J. I. T. H. C. M. B., MA: Jones \& Learning B., (2017). Quality Improvement Basics.

[23] Mullennix, S. C., Iseler, J., Kwiatkowski, G. M., MccannSpry, L., Skinner, J., Kuhl, N., Vandepol, E. K., \& Poland, C. A. J. C. N. S. (2020). A clinical nurse specialist-led emergency department naloxone distribution program. 34, 116-123.

[24] Oakes, L. L., Anghelescu, D. L., Windsor, K. B., Barnhill, P. D. J. J. O. P., \& Management, S. (2008). An institutional quality improvement initiative for pain management for pediatric cancer inpatients. 35, 656-669.

[25] Meissner, W., Huygen, F., Neugebauer, E. A., Osterbrink, J., Benhamou, D., Betteridge, N., Coluzzi, F., De Andres, J., Fawcett, W., Fletcher, D. J. C. M. R., \& Opinion (2018). Management of acute pain in the postoperative setting: the importance of quality indicators. 34, 187-196. 